\title{
Whole-body x-ray dark-field radiography of a human cadaver
}

Jana Andrejewski ${ }^{1 *}$, Fabio De Marco ${ }^{1}$, Konstantin Willer ${ }^{1}$, Wolfgang Noichl' ${ }^{1}$, Alex Gustschin ${ }^{1}$, Thomas Koehler ${ }^{2}$, Pascal Meyer ${ }^{3}$, Fabian Kriner ${ }^{4}$, Florian Fischer ${ }^{4}$, Christian Braunn, Alexander A. Fingerle ${ }^{5}$, Julia Herzen ${ }^{4}$, Franz Pfeiffer ${ }^{1,5}$ and Daniela Pfeiffer ${ }^{5}$

\begin{abstract}
Background: Grating-based x-ray dark-field and phase-contrast imaging allow extracting information about refraction and small-angle scatter, beyond conventional attenuation. A step towards clinical translation has recently been achieved, allowing further investigation on humans.

Methods: After the ethics committee approval, we scanned the full body of a human cadaver in anterior-posterior orientation. Six measurements were stitched together to form the whole-body image. All radiographs were taken at a three-grating large-object x-ray dark-field scanner, each lasting about $40 \mathrm{~s}$. Signal intensities of different anatomical regions were assessed. The magnitude of visibility reduction caused by beam hardening instead of small-angle scatter was analysed using different phantom materials. Maximal effective dose was $0.3 \mathrm{mSv}$ for the abdomen.

Results: Combined attenuation and dark-field radiography are technically possible throughout a whole human body. High signal levels were found in several bony structures, foreign materials, and the lung. Signal levels were $0.25 \pm 0.13$ (mean \pm standard deviation) for the lungs, $0.08 \pm 0.06$ for the bones, $0.023 \pm 0.019$ for soft tissue, and $0.30 \pm 0.02$ for an antibiotic bead chain. We found that phantom materials, which do not produce small-angle scatter, can generate a strong visibility reduction signal.

Conclusion: We acquired a whole-body x-ray dark-field radiograph of a human body in few minutes with an effective dose in a clinical acceptable range. Our findings suggest that the observed visibility reduction in the bone and metal is dominated by beam hardening and that the true dark-field signal in the lung is therefore much higher than that of the bone.
\end{abstract}

Keywords: Dark-field imaging, Human body, Radiography, Whole-body imaging, X-rays

\footnotetext{
* Correspondence: jana.andrejewski@tum.de

${ }^{1}$ Chair of Biomedical Physics, Department of Physics and Munich School of BioEngineering, Technical University of Munich, 85748 Garching, Germany Full list of author information is available at the end of the article
}

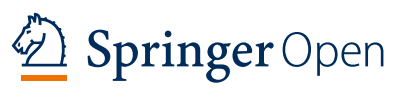

(c) The Author(s). 2021 Open Access This article is licensed under a Creative Commons Attribution 4.0 International License, which permits use, sharing, adaptation, distribution and reproduction in any medium or format, as long as you give appropriate credit to the original author(s) and the source, provide a link to the Creative Commons licence, and indicate if changes were made. The images or other third party material in this article are included in the article's Creative Commons licence, unless indicated otherwise in a credit line to the material. If material is not included in the article's Creative Commons licence and your intended use is not permitted by statutory regulation or exceeds the permitted use, you will need to obtain permission directly from the copyright holder. To view a copy of this licence, visit http://creativecommons.org/licenses/by/4.0/. 


\section{Key points}

- We presented the first whole-body $x$-ray dark-field images of a human body.

- In the used setup, dark-field signal of the bones originates mainly from beam hardening.

- Beam hardening correction methods are important for medical applications of $\mathrm{x}$-ray dark-field radiography.

\section{Background}

Besides the $\mathrm{x}$-ray attenuation, which is measured by conventional $\mathrm{x}$-ray imaging, wave-optical effects such as refraction and small-angle scatter of $\mathrm{x}$-rays occur on interaction with matter.

X-ray phase-contrast imaging utilises a Talbot-Lau interferometer to measure these effects. By placing three gratings in the beam path of a conventional source, intensity modulations are created from which the phase and small-angle scatter information can be obtained. Subsequently, attenuation, differential phase, and darkfield images of a sample placed in the beam path can be calculated form these recorded modulations [1-10]. The dark-field image provides a measure for the magnitude of small-angle $x$-ray scattering induced by a sample. The correlation between dark-field signal and microscopic sample parameters has been examined in detail [11-13].

A conventional source has a broad $x$-ray energy spectrum, and thus, beam hardening occurs when measuring thicker samples. This spectral change also affects the dark-field measurement, as the dark-field signal is highly dependent on the x-ray energy $E[11,13,14]$.

Applications for this new imaging modality have been found both in the context of basic research, nondestructive testing, and medical diagnostics [15-29]. Concerning the latter, the first in vivo dark-field radiograph of a mouse revealed a high dark-field signal originating from the lungs [30], which motivated a large number of small animal studies as many pulmonary diseases are related to structural impairments. These determined a significant potential of dark-field radiography and computed tomography (CT) for the detection of structural pulmonary diseases [31-42]. A further important step towards clinics was the development of $x$-ray dark-field systems allowing imaging studies of the lung of larger animals and human bodies [17, 43-46]. Beyond the lung, the internal structure of bones was also recognised to be a source of dark-field signal [47, 48]. One study [43] presented full body $\mathrm{x}$-ray dark-field and transmission images of a euthanised pig. The authors found strong dark-field signal in the lung as well as in some skeleton structures.

We present transmission and dark-field images covering the majority of a human body. We compare the signal strengths of both types of images for different body regions and discuss the detectability of anomalies on both image modalities. Furthermore, the effect of beam hardening on dark-field images is discussed by comparing the dark-field signal of different scattering and absorbing materials.

\section{Methods}

\section{Imaging setup}

The three-grating setup has been previously described [44-46]. The source grating $\left(G_{0}\right)$ had an area of $5.0 \times$ $2.5 \mathrm{~cm}^{2}$, a period of $68.72 \mu \mathrm{m}$, and a duty cycle of 0.7 . The other two gratings $\left(G_{1}\right.$ : period $8.73 \mu \mathrm{m}$, duty cycle $0.5, G_{2}$ : period $10 \mu \mathrm{m}$, duty cycle 0.5$)$ were created from eight tiles, each with a size of $5.0 \times 2.5 \mathrm{~cm}^{2}$, to achieve a total size of $40 \times 2.5 \mathrm{~cm}^{2}$ per grating, as already described by Schröter et al. [49]. The $G_{0}-G_{1}$ and the $G_{1}-G_{2}$ distances were 1.60 and $0.25 \mathrm{~m}$, respectively. All gratings were gold-filled attenuation gratings (gold filling height of $150-200 \mu \mathrm{m})$ provided by Karlsruhe Institute of Technology (Karlsruhe, Germany) and Microworks $\mathrm{GmbH}$ (Karlsruhe, Germany). For this highly asymmetric configuration, the immediate shadow of $G_{1}$ instead of the Talbot effect was exploited to generate an intensity pattern at the position of $G_{2}$. A similar approach was already described [50]. All gratings are mounted on a common frame pivoted about an axis through the source's focal spot. An effective field of view of $32 \times 35$ $\mathrm{cm}^{2}$ was achieved (in the object plane, $10 \mathrm{~cm}$ above the sample table) by scanning the grating frame across the detector. Source, detector and sample remain stationary during the acquisition. Acquisition time was $40 \mathrm{~s}$ per scan. The x-ray source (Philips MRC 2000310 ROT GS, Philips Medical Systems, Hamburg, Germany) was operated at $70 \mathrm{kVp}$ and $700 \mathrm{~mA}$ for the whole-body measurement.

\section{Data acquisition and processing}

For the acquisition of the individual images, a moiré fringe-scanning method was used [17, 51]. By introducing a slight mismatch of the distance between modulation grating and analyser grating, a low-frequency fringe pattern is generated in the detector plane, representing the relative lateral positions of modulation and analyser grating structures. This pattern is scanned over each sample point via movement of the grating frame, generating a series of images. In this way, a moiré pattern is sampled for each detector pixel, and a method similar to the more common, so-called phase-stepping acquisition procedure $[8,17]$ can be used to retrieve mean intensity $A_{s}$, interferometric visibility $V_{s}$ (i.e., the relative contrast of the fringe pattern), and lateral phase shift $\phi_{s}$ of the pattern. Repeating the procedure without a sample yields the reference values $A_{r}, V_{r}$, and $\phi_{r}$, allowing the 


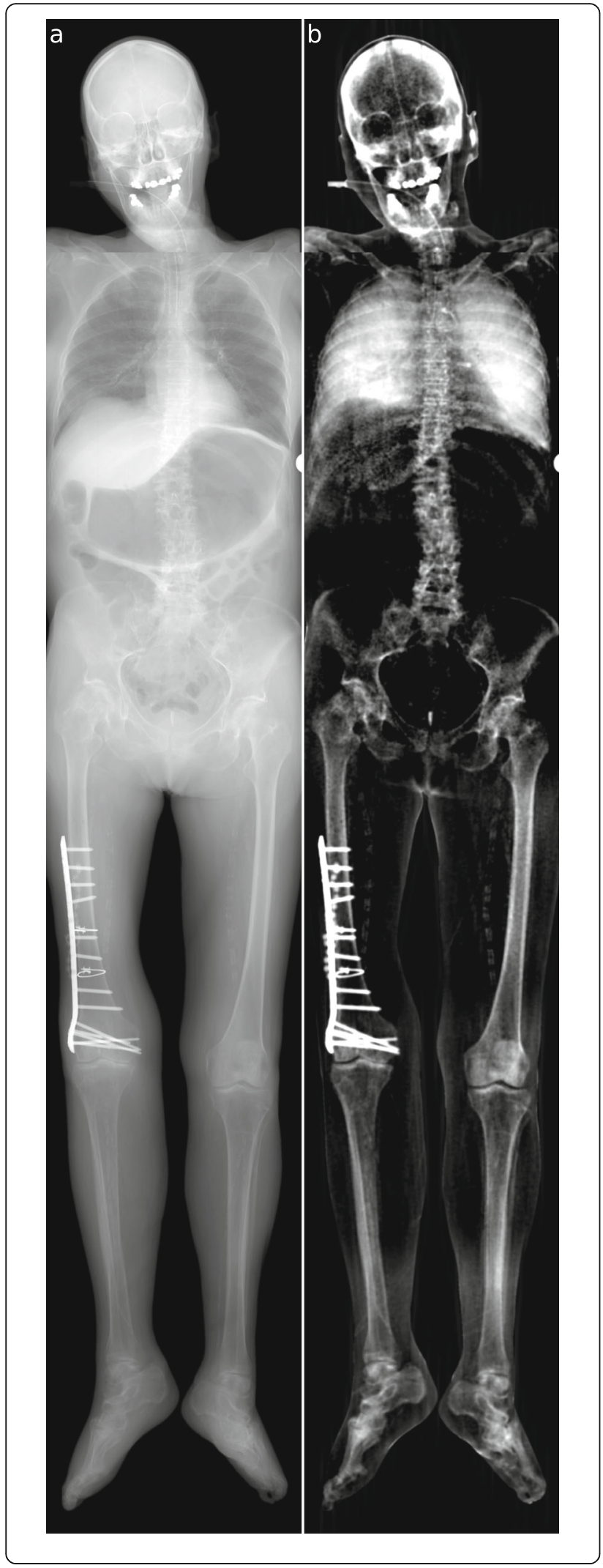

Fig. 1 Attenuation (window level 2.5, window width 5) (a) and x-ray dark-field (window level 0.175, window width 0.35) (b) radiographs of a human cadaver excluding the arms, recumbent, anteroposterior acquisition with the right hand side of the body on the left side. The images are created from six individual scans. A correction for visibility reduction due to beam hardening was applied

calculation of attenuation $\alpha=-\ln \left(A_{s} / A_{r}\right)$ and visibility reduction $v=-\ln \left(V_{s} / V_{r}\right)$ of the sample.

Since a visibility reduction due to beam hardening occurs in areas with high attenuation, a correction method, adapted from Pelzer et al. [52], was used to rectify the dark-field signal: transmission $T_{k}$ and visibility reduction $v_{k}$ signals were measured for several polyoxymethylene (POM) plastic heights $h_{k}$. A reference curve $v_{B H}(T)$, which indicates visibility reduction as a function of transmission, was calculated from these points. The corrected dark-field signal $D$ of a sample is then given by $D=v-v_{B H}$. POM was chosen as a reference material for soft tissue and fat in the visibility reduction calibration as it has similar spectral attenuation properties as soft tissue and fat.

The transmission and dark-field images of the human body were combined from six individual scans, leading to a total scanned area of $177 \times 30 \mathrm{~cm}^{2}$ in the patient plane. The body was placed on a motorised sample table. The upper body was imaged in three scans, and the scan range was selected by adjusting the table position before each scan. Due to the table limited movement range, the body was then rotated by $180^{\circ}$ to allow imaging the lower body. Three additional scans were acquired in this orientation.

\section{Human cadaver}

The experiment was approved by the institutional review board (Ethikkommission der Ludwig-MaximiliansUniversität München, Pettenkoferstraße 8a, 80336 Munich, Germany) and was conducted according to the Declaration of Helsinki. The full-body radiographs were taken from a 62-year-old female body (height $162 \mathrm{~cm}$, weight $49 \mathrm{~kg}$ ). Images were taken 4 days postmortem, and the lung was ventilated by an anaesthesia machine (Fabius ${ }^{\circ}$ Tiro, Drägerwerk AG \& Co. KGaA, Lübeck, Germany) with a constant pressure of 18 mbar.

\section{Dose estimation}

The dose area product was estimated from the incident air kerma, measured with a PTW NOMEX dosimeter (PTW, Freiburg, Germany) and the area of the organ. With this, the effective dose (ED) for different organs can be calculated using conversion factors given by Wall et al. [53]. 


\section{Beam hardening measurements}

In the experiment determining the effect of beam hardening on the $\mathrm{x}$-ray dark-field signal, different heights of POM, aluminium, and neoprene were measured in a phantom experiment as well as an ex situ pig lung obtained from a local butcher. For the values of aluminium, POM and neoprene, the transmission and darkfield signal was calculated from the mean signal across a region of interest of $22 \times 47 \mathrm{~mm}^{2}$ for each material height. The pig lung was ventilated with a pressure of 25 mbar. Each point in the provided scatter plot corresponds to a value pair $(T, v)$ of one pixel in the two (registered) images (see Fig. 4).

\section{Data presentation}

Data are given as simple point values or estimation or as mean \pm standard deviation.

\section{Results}

Full-body transmission and dark-field images of a human body are shown in Fig. 1. These images were combined from six individual scans. Figures 2 and 3 show transmission and dark-field radiographs of the hip and the thigh regions with a narrower windowing in order to highlight features in these regions.

For different organs, a rough estimation of ED is given in Table 1. For comparison, typical ED reference values as reported by Wall et al. [53] are presented for these organs. The ED values for the chest, knee, and feet were a lot higher than the typical ED values, whereas the ED values for the head, abdomen, and pelvis were in the range of the typical ED values.
The inflated lungs exhibit high transparency in the attenuation image. In the dark-field image, both lungs reveal a high signal $(0.25 \pm 0.13)$. The highest signal intensity was seen in the lower lobe of the right lung with 0.34 . Conversely, low transmission signal and no dark-field signal are observed in the abdominal region. Bony structures are visible in the attenuation as well as in the dark-field images. The mean dark-field signal of the bones was $0.08 \pm 0.06$, with a maximum of 0.23 in the cortical bone of the upper left leg. In particular, an increased signal in both modalities occurs in the hip joints, where anatomical changes due to osteoarthritis are present (see Fig. 2). Furthermore, the right leg presents the result of osteosynthesis following a fracture of the right femur. The fracture itself is not visible anymore, which correlates nicely with the CT image, where the complete healing of the fracture can be confirmed. In contrast, the metal screws and plate are clearly visible. In addition, an antibiotic bead chain, which is still in situ, is clearly visible in the dark-field image (0.30 \pm 0.02). With respect to vascular structures, the calcification of the femoral arteries can be clearly depicted in both the upper legs in all three modalities (see Fig. 3). Finally, in soft tissue, barely any dark-field signal can be observed $(0.023 \pm 0.019)$.

Figure 4 shows a plot of uncorrected visibility reduction $v=-\ln \left(V_{s} / V_{r}\right)$ versus the corresponding attenuation signal $\alpha=-\ln \left(A_{s} / A_{r}\right)$ of each measured phantom material (i.e., aluminium, POM, neoprene and an ex situ pig lung). It is apparent that the visibility reduction signal of the lung and neoprene increases more steeply as a function of the transmission signal than that of aluminium. Among all measured materials, the visibility reduction

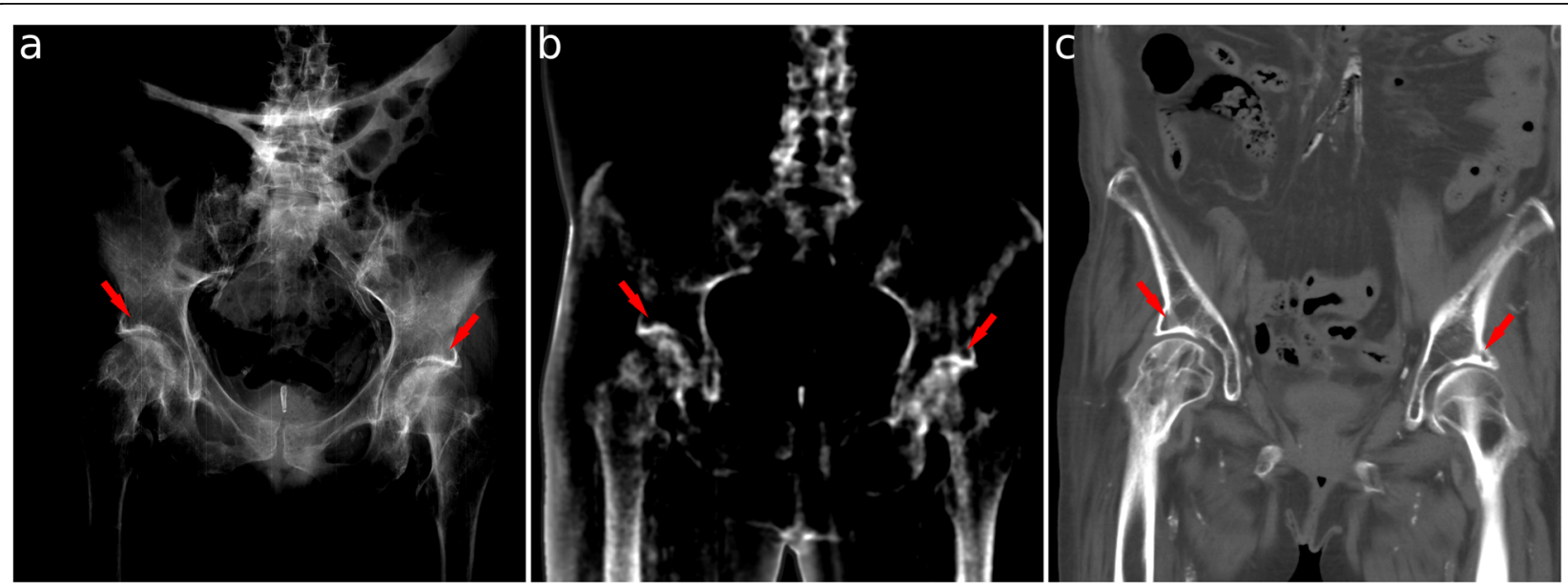

Fig. 2 Conventional x-ray (window level 4.05, window width 1.1) (a) and dark-field radiograph (window level 0.8, window width 2.0) (b), and coronal computed tomography reconstruction (c) of the pelvic region. The red arrows mark an area with subchondral sclerosis as a cause of bilateral osteoarthritis of the hips. These areas exhibit an increased signal at both modalities. Whereas the increased attenuation signal is due to the associated thickening of the bone structure, the increased dark-field signal can be attributed due to spectral hardening effects as well as increased small-angle scatter 


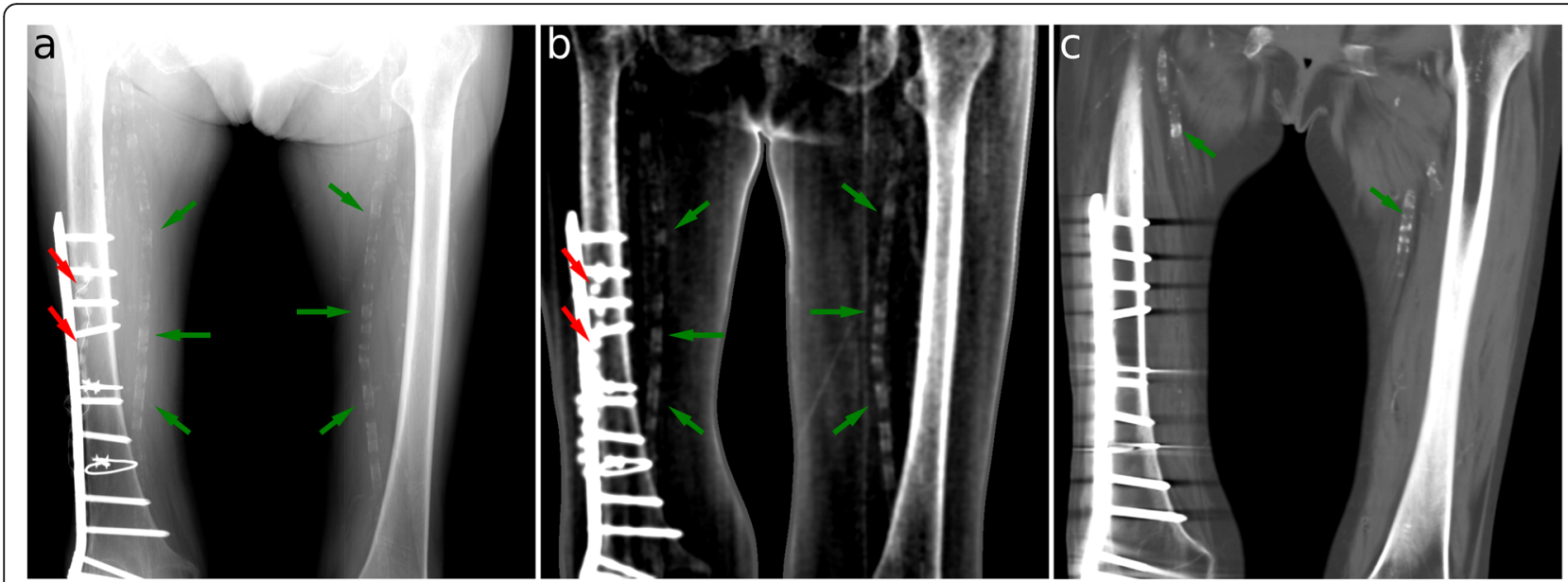

Fig. 3 X-ray attenuation (window level 2.45, window width 1.9) (a), dark-field (window level 0.4, window width 2.0) (b) radiographs, and a computed tomography reconstruction (c) of the upper legs. The metal screws and plate on the right femur, a fixation of an old fracture, are clearly visible in all three modalities. Near the screws, an antibiotic bead chain is also visible in the attenuation and dark-field images (red arrows). Furthermore, calcifications are visible within the superficial femoral arteries in both thighs at all three modalities (green arrows)

signal of POM increases the least with $\alpha$. For POM and aluminium, the transmission signal increases as well as the visibility reduction, even though these objects do not produce small-angle scatter. The increase of visibility reduction is stronger for aluminium than for POM.

\section{Discussion}

In past small animal studies, $\mathrm{x}$-ray dark-field imaging showed a potential benefit in diagnosing lung diseases [31-42, 54]. Subsequent large animal and human cadaver measurements showed the possibility to translate $\mathrm{x}$-ray dark-field radiography to the human scale [43-46], and a first study on dark-field chest radiographs of human bodies showed that the dark-field signal could be reliable quantified [55]. However, further applications of $\mathrm{x}$-ray dark-field imaging has not been extensively investigated on large animal models or human cadavers yet.

In this study, we presented the first $\mathrm{x}$-ray dark-field image of a complete human body and compared findings

Table 1 Rough estimation of effective dose in the study and literature values for different organs

\begin{tabular}{lllr}
\hline Organ & ED $(\mathbf{m S v})$ & Typical ED $(\mathbf{m S v})^{\mathbf{a}}$ & ED/typical ED \\
\hline Head AP & 0.034 & 0.033 & 1.03 \\
Chest PA & 0.268 & 0.014 & 19.14 \\
Abdomen AP & 0.300 & 0.430 & 0.70 \\
Pelvis AP & 0.220 & 0.280 & 0.79 \\
Femur AP & 0.051 & 0.011 & 4.64 \\
Knee AP & 0.004 & 0.0001 & 40.00 \\
Feet oblique & 0.0024 & 0.0001 & 24.00 \\
\hline
\end{tabular}

$A P$ Anteroposterior, $P A$ Posteroanterior, ED Effective dose a Wall et al. [53] in the transmission, dark-field and CT images. A high dark-field signal was found in the lung. Furthermore, the bone, calcification in the femoral arteries, implants and foreign bodies also produce a dark-field signal. Phantom measurements showed that a strong visibility reduction signal can be produced by objects which do not generate small-angle scatter.

In this study, the ED values resulted to be higher than typical ED in some organs. The typical ED are given for radiographs where only the organ in question is imaged. As a consequence, image parameters like tube current were chosen to be optimal for imaging this organ. In our study, the imaging parameters were the same for all organs. Thus, the tube current had to be chosen in such a way that enough photons reach the detector behind the more absorbing body parts like head, pelvis, and abdomen. For those regions, the ED in this study resulted to be similar to the typical ED. Higher ED in less absorbing organs is a consequence of this experimental setup.

Our results concerning the thorax region are similar to the results reported in other studies $[43,44,46]$. The considerable dark-field signal in the lung originates from small-angle scattering on the numerous air-tissue interfaces in the lung parenchyma, whereas the high transmission is due to the weak attenuation of the mainly airfilled lungs. In this study, the dark-field signal of the lung was lower compared to the dark-field signal of the pig lung reported by Hauke et al. [43]. The authors euthanised a pig a few minutes before image acquisition, whereas in this study, the images were taken 4 days postmortem. Therefore, the lung of the human body was more strongly collapsed with partially fluid-filled alveoli compared to the pig lung and not as many air-tissue interfaces were present anymore in the human lung. 


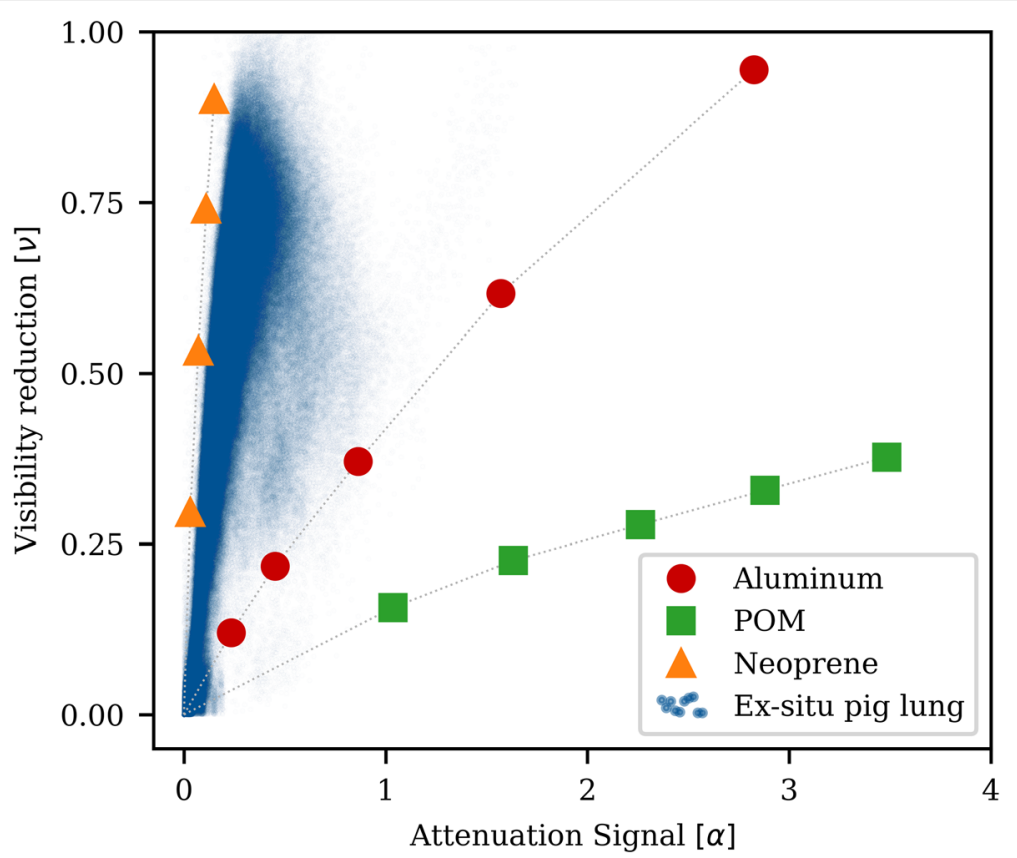

Fig. 4 Effect of beam hardening and small-angle scattering on the x-ray dark-field signal. Measured with 1, 2, 4, 8, and 16 mm of aluminium; 3, 5, 7,9 , and $11 \mathrm{~cm}$ of polyoxymethylene (POM); 1,2,3, and $4 \mathrm{~cm}$ of neoprene and an ex situ pig lung. Although no scattering object was in the beam path during the measurement of POM and aluminium, an apparent dark-field signal was generated by beam hardening. Aluminium exhibits similar spectral attenuation properties as the bone, whereas POM more closely approximates soft and adipose tissue. The large discrepancy between the two curves explains the strong apparent dark-field signal due to the bones in Figs. 1, 2, and 3, despite application of a POM-based beam hardening correction

In contrast to the lungs, barely any air-tissue interfaces exist in the stomach and, as a consequence, no darkfield signal originates in the stomach. The low attenuation signal is a result of the stomach being extensively filled with air due to a previous incorrect positioning of the endotracheal tube within the oesophagus.

Dark-field signal is generated by the individual beads of the antibiotic bead chain, as these are made from a spongious material which acts as a substrate for the antibiotic agent. Spongious materials contain large numbers of interfaces with air, leading to an increase in scattering and therefore to an increased dark-field signal.

Calcification, i.e., accumulation of calcium salt, in particular of arterial walls (as it can be seen in atherosclerosis), is a common finding, especially in patients with cardiovascular risk factors. Atherosclerosis can lead to stenosis of the affected arteries, resulting in hypoperfusion of the anatomical region which is supplied by the respective artery. Depending on the affected arteries, patients can suffer from coronary or peripheral artery disease, as it was for the patient in our study. Previous studies showed that calcification in the breast is visible in the dark-field image, potentially improving breast cancer detection [56-58]. We were also able to see calcification of the arteries in the dark-field image. However, whether the calcification is better and earlier visible on dark-field than common transmission images and if dark-field imaging has the potential to improve the diagnostic value has to be determined in further studies.

Bony structures are visible in both transmission and dark-field images, especially the areas of increased calcium content: subchondral sclerosis as a cause of bilateral osteoarthritis of the hips was highly visible in the dark-field image. Similarly, subchondral sclerosis due to osteoarthritis of both knees also resulted in a high signal on the dark-field images.

As visible in Fig. 4, even non-scattering materials can cause a reduction in visibility which is due to beam hardening. The interferometric visibility of an $\mathrm{x}$-ray dark-field imaging setup is not only dependent on the setup arrangement (i.e., grating periods and inter-grating distances), but also on the spectrum of the x-ray beam. In our setup, visibility was high for photon energies up to $40 \mathrm{keV}$ and decreases continuously for higher energies. When measuring with energy integrating detectors, the average visibility results from the detector signal of the energy dependent visibility weighted with the detected signal from each photon energy [59]. Since the low-energy x-rays, which contribute the most to the measured visibility, are preferentially attenuated by most materials, the measured, average visibility decreases. As a result, non-scattering, strongly attenuating materials can induce a decrease of visibility, which is 
indistinguishable from a true dark-field signal due to small-angle $\mathrm{x}$-ray scatter. For soft and adipose tissues, this effect could largely be eliminated by a correction, which is based on visibility reduction measurements of POM. As POM has spectral attenuation properties similar to soft tissue, which differs significantly from those of bone and metal, the correction however fails to accurately model their contribution to beam hardening, and a residual visibility reduction signal remains.

It has been shown that the bones also generate a darkfield signal $[47,48,60]$. The dark-field signal of the bones is lower as the signal of the lungs. Our setup showed a lower sensitivity than the ones used for bone measurements. Therefore, in our opinion, the dark-field signal of the bones in the here presented measurements mainly result from residual visibility reduction signal.

Our study has some limitations. First, only one human body was imaged. Furthermore, the complete image was stitched together from six individual scans and the body had to be repositioned during the acquisition procedure. This experimental setup is not practical as an imaging approach in clinical routine. This problem could be overcome by changing the acquisition of the dark-field images: instead of moving the interferometer, the sample could be moved as proposed by Seifert et al. [61]. Thus, the patient can be imaged in one scan similar to a full body CT scan. Lastly, the beam hardening correction was performed with respect to the soft tissue component. Removal of beam-hardening-related artefact depends on the knowledge of the material composition of the body part in question. Calibration data and algorithms used for correction must therefore be tailored to the measured body part. In particular, superposition of materials with very different spectral properties (e.g., bone and soft tissue) presents a special challenge for beam-hardening-related visibility correction in dark-field radiography, since the individual material contributions to the total attenuation cross-section along the $\mathrm{x}$-ray path are unknown and vary over the field of view. A useful approach in such a case may be to use dual-energy acquisition, which may allow calculating spatial maps of such cross-section fractions and thus enabling correction of beam-hardening-related dark-field artefact for two different materials.

In conclusion, we gave an overview over the dark-field signal strength of different organs of the human body presenting images and data from a female cadaver. However, before dark-field imaging can be included into the clinical routine, further studies will have to be conducted. Besides lung imaging, bone imaging and the possibility of diagnosing calcification might be of special interest.
Abbreviations

CT: Computed tomography; ED: Effective dose; POM: Polyoxymethylene

\section{Acknowledgements}

This work was carried out with the support of the Karlsruhe Nano Micro Facility (KNMF, www.kit.edu/knmf), a Helmholtz Research Infrastructure at Karlsruhe Institute of Technology (KIT).

We acknowledge the financial support through the European Research Council (AdG 695045) and the DFG (GRK 2274).

\section{Authors' contributions}

$J A, F D M, K W, F K, F F$, and CB performed the experiments. FDM, KW, WN, AG, $T K, P M, J H$, and FP developed the hardware, the control software and the data processing algorithms of the imaging system. JA, FDM, and DP performed the data analysis. AAF, JH, FP, and DP supervised the project. All authors reviewed the manuscript. The authors read and approved the final manuscript.

\section{Funding}

European Research Council (AdG 695045) and DFG (GRK 2274). Open Access funding enabled and organised by Projekt DEAL.

Availability of data and materials

The datasets used and/or analysed during the current study are available from the corresponding author on reasonable request.

\section{Ethics approval and consent to participate}

The experiment was approved by the institutional review board (Ethikkommission der Ludwig-Maximilians-Universität München, Pettenkoferstr. 8a, 80336 Munich, Germany) and was conducted according to the Declaration of Helsinki.

\section{Consent for publication}

Not applicable

\section{Competing interests}

One of the authors of this manuscript (Thomas Koehler) is an employee of Philips. The remaining authors declare that they have no competing interests.

\section{Author details}

${ }^{1}$ Chair of Biomedical Physics, Department of Physics and Munich School of BioEngineering, Technical University of Munich, 85748 Garching, Germany. ${ }^{2}$ Philips Research, 22335 Hamburg, Germany. ${ }^{3}$ Institute of Microstructure Technology, Karlsruhe Institute of Technology, 76344

Eggenstein-Leopoldshafen, Germany. ${ }^{4}$ Institut für Rechtsmedizin, Ludwig-Maximilians-Universität München, 80336 Munich, Germany.

${ }^{5}$ Department of Diagnostic and Interventional Radiology, Technical University of Munich, 81675 Munich, Germany.

Received: 22 July 2020 Accepted: 3 December 2020

Published online: 26 January 2021

\section{References}

1. Bonse U, Hart M (1965) An x-ray interferometer. Appl Phys Lett 6:155-156. https://doi.org/10.1063/1.1754212

2. Morrison GR, Browne MT (1992) Dark-field imaging with the scanning transmission x-ray microscope. Rev Sci Instrum 63:611-614. https://doi.org/ 10.1063/1.1143820

3. Ingal VN, Beliaevskaya EA (1995) X-ray plane-wave topography observation of the phase contrast from a non-crystalline object. J Phys D Appl Phys 28 : 2314-2317. https://doi.org/10.1088/0022-3727/28/11/012

4. Davis TJ, Gao D, Gureyev TE, Stevenson AW, Wilkins SW (1995) Phasecontrast imaging of weakly absorbing materials using hard X-rays. Nature 373:595-598. https://doi.org/10.1038/373595a0

5. Snigirev A, Snigireva I, Kohn V, Kuznetsov S, Schelokov I (1995) On the possibilities of $x$-ray phase contrast microimaging by coherent high-energy synchrotron radiation. Rev Sci Instrum 66:5486-5492. https://doi.org/10. $1063 / 1.1146073$ 
6. Wilkins SW, Gureyev TE, Gao D, Pogany A, Stevenson AW (1996) Phasecontrast imaging using polychromatic hard X-rays. Nature 384:335-338. https://doi.org/10.1038/384335a0

7. Momose A, Kawamoto S, Koyama I, Hamaishi Y, Takai K, Suzuki Y (2003) Demonstration of X-ray Talbot Interferometry. Jpn J Appl Phys 42(7B):L866L868. https://doi.org/10.1143/JJAP.42.L866

8. Weitkamp T, Diaz A, David C et al (2005) X-ray phase imaging with a grating interferometer. Opt Express 13:6296-6304. https://doi.org/10.1364/ OPEX.13.006296

9. Pfeiffer F, Weitkamp T, Bunk O, David C (2006) Phase retrieval and differential phase-contrast imaging with low-brilliance X-ray sources. Nat Phys 2:258-261. https://doi.org/10.1038/nphys265

10. Pfeiffer F, Bech M, Bunk $\mathrm{O}$, et al (2008) Hard-X-ray dark-field imaging using a grating interferometer. Nat Mater 7:134-137. https://doi.org/10.1038/ nmat2096

11. Lynch SK, Pai V, Auxier J et al (2011) Interpretation of dark-field contrast and particle-size selectivity in grating interferometers. Appl Opt 50:4310-4319. https://doi.org/10.1364/AO.50.004310

12. Strobl M (2014) General solution for quantitative dark-field contrast imaging with grating interferometers. Sci Rep 4:7243 https://doi.org/10.1038/srep07243

13. Yashiro W, Terui Y, Kawabata K, Momose A (2010) On the origin of visibility contrast in x-ray Talbot interferometry. Opt Express 18:16890-16901. https:// doi.org/10.1364/OE.18.016890

14. Attwood D (1999) Soft X-rays and extreme ultraviolet radiation. Cambridge University Press, Cambridge. https://doi.org/10.1017/CBO9781139164429

15. Chabior M, Donath T, David C, et al (2011) Signal-to-noise ratio in $x$ ray dark-field imaging using a grating interferometer. J Appl Phys 110:053105. https://doi.org/10.1063/1.3630051

16. Donath T, Chabior M, Pfeiffer $F$ et al (2009) Inverse geometry for gratingbased $x$-ray phase-contrast imaging. J Appl Phys 106:054703. https://doi. org/10.1063/1.3208052

17. Kottler C, Pfeiffer F, Bunk O, Grünzweig C, David C (2007) Grating interferometer based scanning setup for hard $x$-ray phase contrast imaging. Rev Sci Instrum 78:043710. https://doi.org/10.1063/1.2723064

18. Prade F, Fischer K, Heinz D, Meyer P, Mohr J, Pfeiffer F (2016) Time resolved $X$-ray dark-field tomography revealing water transport in a fresh cement sample. Sci Rep 6:29108. https://doi.org/10.1038/srep29108

19. Revol V, Plank B, Kaufmann R, Kastner J, Kottler C, Neels A (2013) Laminate fibre structure characterization of carbon fibre-reinforced polymers by $X$-ray scatter dark field imaging with a grating interferometer. NDT E Int 58:64-71. https://doi.org/10.1016/j.ndteint.2013.04.012

20. Bachche S, Nonoguchi M, Kato K et al (2017) Laboratory-based X-ray phaseimaging scanner using Talbot-Lau interferometer for non-destructive testing. Sci Rep 7:6711. https://doi.org/10.1038/s41598-017-07032-y

21. Braig E, Birnbacher $L$, Schaff $F$ et al (2018) Simultaneous wood and metal particle detection on dark-field radiography. Eur Radiol Exp 2:1. https://doi. org/10.1186/s41747-017-0034-1

22. Braig E, Roiser N, Kimm MA et al (2020) X-ray dark-field radiography - potential for visualization of monosodium urate deposition. Invest Radiol 55:494-498. https://doi.org/10.1097/rli.0000000000000671

23. Horn F, Gelse K, Jabari S et al (2017) High-energy x-ray Talbot-Lau radiography of a human knee. Phys Med Biol 62:6729-6745. https://doi.org/ 10.1088/1361-6560/aa7721

24. Arboleda C, Wang Z, Jefimovs K et al (2019) Towards clinical gratinginterferometry mammography. Eur Radiol 30:1419-1425. https://doi.org/10, 1007/s00330-019-06362-x

25. Eggl E, Grandl S, Sztrókay-Gaul A et al (2018) Dose-compatible gratingbased phase-contrast mammography on mastectomy specimens using a compact synchrotron source. Sci Rep 8:15700. https://doi.org/10.1038/ s41598-018-33628-z

26. Velroyen A, Bech M, Malecki A et al (2013) Microbubbles as a scattering contrast agent for grating-based $x$-ray dark-field imaging. Phys Med Biol 58: N37-N46. https://doi.org/10.1088/0031-9155/58/4/N37

27. De Marco F, Willer K, Gromann LB et al (2019) Contrast-to-noise ratios and thickness-normalized, ventilation-dependent signal levels in dark-field and conventional in vivo thorax radiographs of two pigs. PLoS One 14(6): e0217858. https://doi.org/10.1371/journal.pone.0217858

28. Gradl R, Morgan KS, Dierolf $M$ et al (2018) Dynamic in vivo chest $x$-ray darkfield imaging in mice. IEEE Trans Med Imaging 38:649-656. https://doi.org/ 10.1109/tmi.2018.2868999
29. Michel R, Rieger J, Anton $\mathrm{G}$ et al (2013) On a dark-filed signal generated by micrometer-sized calcifications in phase-contrast mammography. Phys Med Biol 58:2713-2732. https://doi.org/10.1088/0031-9155/58/8/2713

30. Bech M, Tapfer A, Velroyen A et al (2013) In-vivo dark-field and phasecontrast x-ray imaging. Sci Rep 3:3209. https://doi.org/10.1038/srep03209

31. Schleede S, Meinel FG, Bech M et al (2012) Emphysema diagnosis using Xray dark-field imaging at a laser-driven compact synchrotron light source. Proc Natl Acad Sci USA 109:17880-17885. https://doi.org/10.1073/pnas. 1206684109

32. Yaroshenko A, Meinel FG, Bech M et al (2013) Pulmonary emphysema diagnosis with a preclinical small-animal X-ray dark-field scatter-contrast scanner. Radiology 269:427-433. https://doi.org/10.1148/radiol.13122413

33. Meinel FG, Yaroshenko A, Hellbach K et al (2014) Improved diagnosis of pulmonary emphysema using in vivo dark-field radiography. Invest Radiol 49:653-658. https://doi.org/10.1097/rli.0000000000000067

34. Hellbach K, Yaroshenko A, Meinel FG et al (2015) In vivo dark-field radiography for early diagnosis and staging of pulmonary emphysema. Invest Radiol 50:430-435. https://doi.org/10.1097/rli.0000000000000147

35. Yaroshenko A, Hellbach K, Yildirim ÄO et al (2015) Improved In vivo Assessment of Pulmonary Fibrosis in Mice using X-Ray Dark-Field Radiography. Sci Rep 5:17492. https://doi.org/10.1038/srep17492

36. Hellbach K, Yaroshenko A, Willer K et al (2016) Facilitated diagnosis of pneumothoraces in newborn mice using $X$-ray dark-field radiography. Invest. Radiol. 51(10):597-601. https://doi.org/10.1097/rli.0000000000000285

37. Yaroshenko A, Pritzke T, Koschlig M et al (2016) Visualization of neonatal lung injury associated with mechanical ventilation using $x$-ray dark-field radiography. Sci Rep 6:24269. https://doi.org/10.1038/srep24269

38. Scherer K, Yaroshenko A, Bölükbas DA et al (2017) X-ray dark-field radiography-in-vivo diagnosis of lung cancer in mice. Sci Rep 7:402. https://doi.org/10.1038/s41598-017-00489-x

39. Hellbach K, Meinel FG, Conlon TM et al (2018) X-ray dark-field imaging to depict acute lung inflammation in mice. Sci Rep 8:2096. https://doi.org/10 1038/s41598-018-20193-8

40. Velroyen A, Yaroshenko A, Hahn D et al (2015) Grating-based X-ray darkfield computed tomography of living mice. EBioMedicine 2:1500-1506. https://doi.org/10.1016/..ebiom.2015.08.014

41. Meinel FG, Schwab, F, Yaroshenko A et al (2014) Lung tumors on multimodal radiographs derived from grating-based X-ray imaging - a feasibility study. Phys Med 30:252-257. https://doi.org/10.1016/j.ejmp.2013. 11.001

42. Meinel F, Schwab, F, Schleede S et al (2013) Diagnosing and mapping pulmonary emphysema on $\mathrm{X}$-ray projection images: incremental value of grating-based X-ray dark-field imaging. PLoS One 8:e59526. https://doi.org/ 10.1371/journal.pone.0059526

43. Hauke C, Bartl P, Leghissa M et al (2018) A preclinical Talbot-Lau prototype for x-ray dark-field imaging of human-sized objects. Med Phys 45:25652571. https://doi.org/10.1002/mp.12889

44. Gromann LB, De Marco F, Willer K et al (2017) In-vivo X-ray dark-field chest radiography of a pig. Sci Rep 7:4807. https://doi.org/10.1038/s41598-01705101-w

45. Hellbach K, Baehr A, De Marco F et al (2018) Depiction of pneumothoraces in a large animal model using $x$-ray dark-field radiography. Sci Rep 8:2602 https://doi.org/10.1038/s41598-018-20985-y

46. Willer K, Fingerle AA, Gromann LB et al (2018) X-ray dark-field imaging of the human lung - a feasibility study on a deceased body. PLoS One 13: e0204565. https://doi.org/10.1371/journal.pone.0204565

47. Eggl E, Malecki A, Schaff $F$ et al (2015) Prediction of vertebral failure load by using X-ray vector radiographic imaging. Radiology 275:553-561. https://doi. org/10.1148/radiol.14141317

48. Baum T, Eggl E, Malecki A et al (2015) X-ray dark-field vector radiography-a novel technique for osteoporosis imaging. J Comput Assist Tomogr 39:286-289. https://doi.org/10.1097/rct.0000000000000192

49. Schröter TJ, Koch FJ, Meyer P et al (2917) Large field-of-view tiled grating structures for X-ray phase-contrast imaging. Rev Sci Instrum 88:015104. https://doi.org/10.1063/1.4973632

50. Huang ZF, Kang KJ, Zhang L et al (2009) Alternative method for differential phase-contrast imaging with weakly coherent hard $x$ rays. Phys Rev A 79: 013815. https://doi.org/10.1103/PhysRevA.79.013815

51. Koehler T, Daerr H, Martens $G$ et al (2015) Slit-scanning differential x-ray phase-contrast mammography: proof-of-concept experimental studies. Med Phys 42:1959-1965. https://doi.org/10.1118/1.4914420 
52. Pelzer $\mathrm{G}$, Anton $\mathrm{G}$, Horn $\mathrm{F}$ et al (2016) A beam hardening and dispersion correction for x-ray dark-field radiography. Med Phys 43:2774-2779. https:// doi.org/10.1118/1.4948671

53. Wall BF, Haylock R, Jansen JTM, Hillier MC, Hart D, Shrimpton PC (2011) Radiation risks from medical $X$-ray examinations as a function of the age and sex of the patient. Health Protection Agency, Centre for Radiation, Chemical and Environmental Hazards. https://www.gov.uk/government/ publications/medical-x-rays-radiation-risks-by-age-and-sex-of-patient

54. Burkhardt R, Gora T, Fingerle AA et al (2020) Early detection of radiationinduced lung damage with $\mathrm{X}$-ray dark-field radiography in mice. Eur Radiol. https://doi.org/10.1007/s00330-020-07459-4

55. Fingerle, AA, De Marco F, Andrejewski, J et al (2019) Imaging features in post-mortem $x$-ray dark-field chest radiographs and correlation with conventional x-ray and CT. Eur Radiol Exp 3, 25. https://doi.org/10.1186/ s41747-019-0104-7

56. Scherer K, Braig E, Ehn S, (2016) Improved diagnostics by assessing the micromorphology of breast calcifications via X-ray dark-field radiography. Sci Rep 6:36991. https://doi.org/10.1038/srep36991

57. Rauch T, Rieger J, Pelzer $\mathrm{G}$ et al (2020) Discrimination analysis of breast calcifications using x-ray dark-field radiography. Med Phys 47:1813-1826. https://doi.org/10.1002/mp.14043

58. Li, X., Gao, H., Chen, Z. et al. (2018) Diagnosis of breast cancer based on microcalcifications using grating-based phase contrast CT. Eur Radiol 28, 3742-3750. https://doi.org/10.1007/s00330-017-5158-4

59. Yashiro W, Vagovič $P$, Momose A (2015) Effect of beam hardening on a visibility-contrast image obtained by X-ray grating interferometry. Opt Express 23:23462-23471. https://doi.org/10.1364/OE.23.023462

60. Jud C, Braig E, Dierolf M et al (2017) Trabecular bone anisotropy imaging with a compact laser-undulator synchrotron x-ray source. Sci Rep 7:14477. https://doi.org/10.1038/s41598-017-14830-x

61. Seifert M, Ludwig V, Kaeppler S et al (2019) Talbot-Lau x-ray phase-contrast setup for fast scanning of large samples. Sci Rep 9:4199. https://doi.org/10. 1038/s41598-018-38030-3

\section{Publisher's Note}

Springer Nature remains neutral with regard to jurisdictional claims in published maps and institutional affiliations.

\section{Submit your manuscript to a SpringerOpen ${ }^{\circ}$ journal and benefit from:}

- Convenient online submission

- Rigorous peer review

- Open access: articles freely available online

High visibility within the field

- Retaining the copyright to your article

Submit your next manuscript at $\boldsymbol{\nabla}$ springeropen.com 